Article

\title{
Beckmann Rearrangement of Ketoxime Catalyzed by $N$-methyl-imidazolium Hydrosulfate
}

\author{
Hongyu $\mathrm{Hu}^{\dagger}{ }^{+}$, Xuting Cai ${ }^{\dagger}$, Zhuying Xu, Xiaoyang Yan * and Shengxian Zhao * \\ Xingzhi College, Zhejiang Normal University, Jinhua 321004, China; huhongyu22@126.com (H.H.); \\ CxuT1998@163.com (X.C.); hu841124@126.com (Z.X.) \\ * Correspondence: eastmorningsun@163.com (X.Y.); shengxian.zhao@apeloa.com (S.Z.); \\ Tel./Fax: +86-579-8229-1129 (X.Y. \& S.Z.) \\ + These authors contributed equally to this work.
}

Received: 7 June 2018; Accepted: 14 July 2018; Published: 18 July 2018

check for updates

\begin{abstract}
Beckmann rearrangement of ketoxime catalyzed by acidic ionic liquid- $N$-methylimidazolium hydrosulfate was studied. Rearrangement of benzophenone oxime gave the desirable product with $45 \%$ yield at $90{ }^{\circ} \mathrm{C}$. When co-catalyst $\mathrm{P}_{2} \mathrm{O}_{5}$ was added, the yield could be improved to $91 \%$. The catalyst could be reused three cycles with the same efficiency. Finally, reactions of other ketoximes were also investigated.
\end{abstract}

Keywords: Beckmann rearrangement; ketoxime; acidic ionic liquid; catalysis

\section{Introduction}

Over the past years, amide derivatives have received much attention owing to their broad range of applications in many fields such as the pharmaceutical industry, chemical biology, the agrochemical industry, engineering plastics, and so on [1-6]. Various approaches have been developed for the synthesis of amide compounds including nucleophilic acyl substitution reactions with amines [7], Staudinger ligation [8], Schmidt reaction [9] and Beckmann rearrangement [10]. However, generations of large amounts of undesired by-products and corrosive phenomenon associated with common acid $\left(\mathrm{H}_{2} \mathrm{SO}_{4}\right.$ and $\left.\mathrm{SOCl}_{2}\right)$ based on liquid phase protocols provide a challenging task for chemists to develop alternative methods [11,12]. A variety of alternative routes [13-16] based on organic and inorganic solid acids were developed. However, traditional methods often suffer from some drawbacks such as poor selectivity, harsh conditions, are not atom economic, or are not environmentally friendly.

From the point of view of atom conversion efficiency, Beckmann rearrangement is a perfect way for construction of amides, in general sulfuric acid is most commonly used rearrangement catalyst in commercial production of amides. However, it brings equipment corrosion and environmental pollution problems. Recently, ionic liquids [17] have emerged as potential green alternatives to organic solvents due to their unique properties of low volatility, high polarity, good thermal stability, and excellent solubility [18-20]. Further, there are more potential capabilities as effective catalysts and reagents [13], as chemical transformations have also been explored. In order to develop a green pathway of amide synthesis, we report here a Beckmann rearrangement reaction catalyzed by $N$-methyl-imidazolium hydrosulfate $\left([\mathrm{HMIm}] \mathrm{HSO}_{4}\right)[21]$ under solvent free conditions (Scheme 1). 
<smiles>[R]NC([R])=O</smiles>

Scheme 1. Synthesis of amides. Reagents and conditions: (a) $\mathrm{NH}_{2} \mathrm{OH} . \mathrm{HCl}, \mathrm{NaOH}, \mathrm{EtOH}, \mathrm{H}_{2} \mathrm{O}$, reflux; (b) acidic ionic liquid, $\mathrm{P}_{2} \mathrm{O}_{5}, \mathrm{~N}_{2}, 90^{\circ} \mathrm{C}, 6 \mathrm{~h}$.

\section{Results}

Beckmann rearrangement of benzophenone oxime catalyzed by $\left[\mathrm{HMIm} \mathrm{HSO}_{4}\right.$ was carried out at $120^{\circ} \mathrm{C}$ over $6 \mathrm{~h}$ without any solvent, the desired product, benzanilide, was obtained in moderate yield (45\%). The co-catalysts such as $\mathrm{P}_{2} \mathrm{O}_{5}, \mathrm{FeCl}_{3}, \mathrm{ZnCl}_{2}, \mathrm{CuCl}_{2} \cdot 2 \mathrm{H}_{2} \mathrm{O}$, and $\mathrm{AlCl}_{3}$ were investigated in this reaction system, the yield was improved significantly to $91 \%$ with $\mathrm{P}_{2} \mathrm{O}_{5}$. However, it has been shown in the literature that the conversion is around $20 \%$ only when $\mathrm{P}_{2} \mathrm{O}_{5}$ is used as the sole catalyst of Beckmann rearrangement [15]. When $\mathrm{CuCl}_{2} \cdot 2 \mathrm{H}_{2} \mathrm{O}$ was added, the yield was reduced to $14 \%$, and the reverse reaction of benzophenone oxime was observed, benzophenone was regenerated. The results are presented in Table 1.

Table 1. Effect of co-catalyst on Beckmann rearrangement in ionic liquid systems.

\begin{tabular}{ccc}
\hline Entry & Co-Catalyst & Yield (\%) \\
\hline 1 & $\mathrm{a}$ & 45 \\
2 & $\mathrm{P}_{2} \mathrm{O}_{5}$ & 91 \\
3 & $\mathrm{FeCl}_{3}$ & 47 \\
4 & $\mathrm{AlCl}_{3}$ & 50 \\
5 & $\mathrm{ZnCl}_{2}$ & 53 \\
6 & $\mathrm{CuCl}_{2} \cdot 2 \mathrm{H}_{2} \mathrm{O}$ & $14{ }^{\mathrm{b}}$
\end{tabular}

Reaction conditions: Benzophenone oxime (9.5 mmol), [HMIm] $\mathrm{HSO}_{4}(11.4 \mathrm{mmol})$, and co-catalyst $(8 \%), 90{ }^{\circ} \mathrm{C}, 6 \mathrm{~h}$;

${ }^{\mathrm{a}}$ without co-catalyst; ${ }^{\mathrm{b}}$ benzophenone $(\%)$ was obtained.

The effect of the amount of co-catalyst $\mathrm{P}_{2} \mathrm{O}_{5}$ on reaction was investigated. The reaction yield was improved when more co-catalyst was added, and the best yield was around $90 \%$ when the amount of $\mathrm{P}_{2} \mathrm{O}_{5}$ was higher than $8 \mathrm{~mol} \%$. The results are presented in Figure 1.

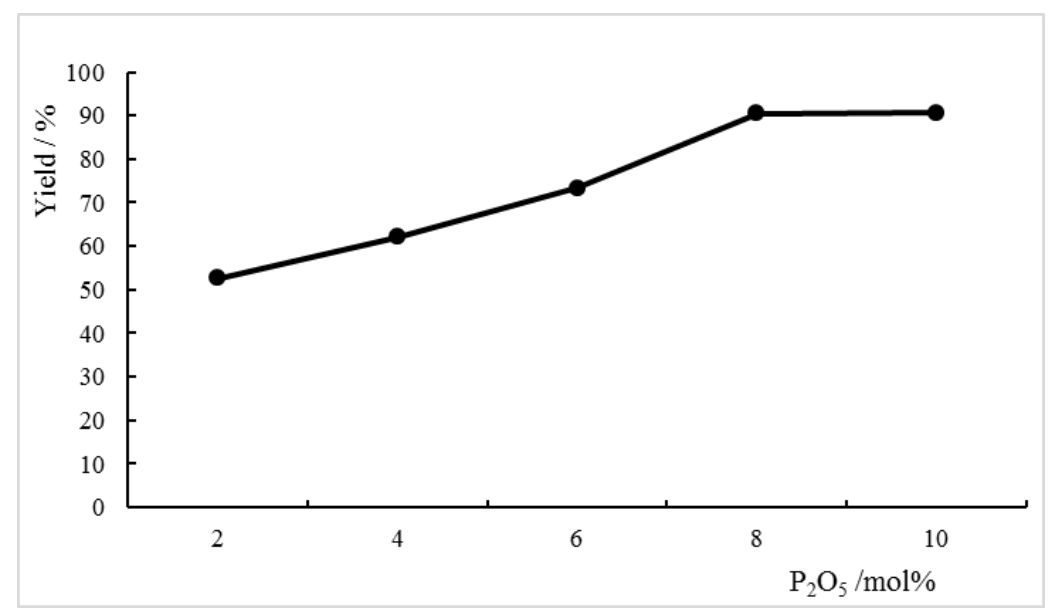

Figure 1. Effect of the amount of co-catalyst $\mathrm{P}_{2} \mathrm{O}_{5}$ on Beckmann rearrangement of benzophenone oxime. Reaction conditions: Benzophenone oxime $(9.5 \mathrm{mmol}),[\mathrm{HMIm}] \mathrm{HSO}_{4}(11.4 \mathrm{mmol}), 90{ }^{\circ} \mathrm{C}, 6 \mathrm{~h}$. 
The influence of the reaction temperature on the yield was investigated subsequently. It was found that $90{ }^{\circ} \mathrm{C}$ is the best reaction temperature. The results are presented in Figure 2.

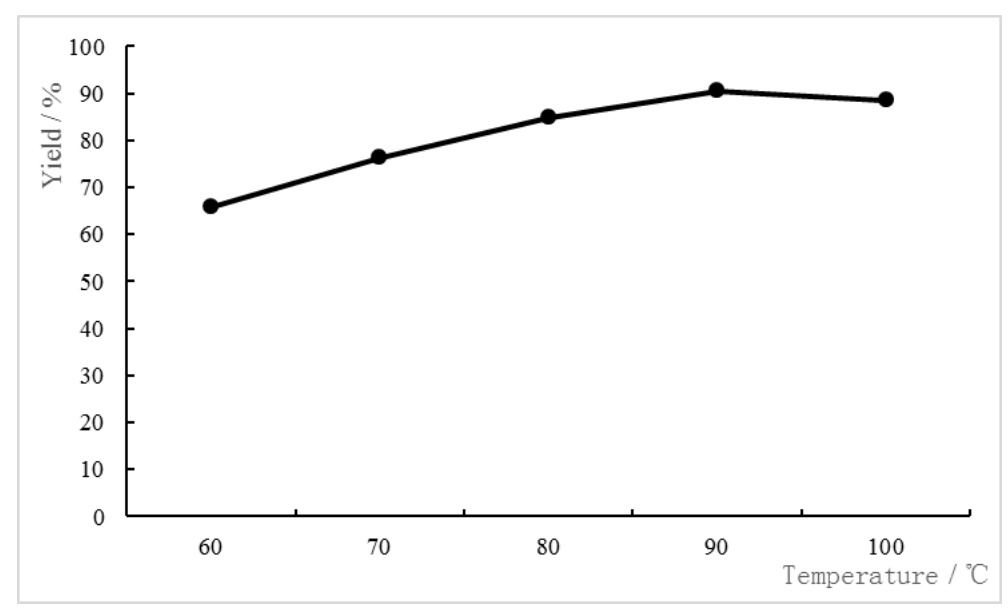

Figure 2. Influence of the reaction temperature on Beckmann rearrangement of benzophenone oxime. Reaction conditions: Benzophenone oxime (9.5 mmol), [HMIm] $\mathrm{HSO}_{4}(11.4 \mathrm{mmol}), 6 \mathrm{~h}$, co-catalyst $\left(\mathrm{P}_{2} \mathrm{O}_{5} 8 \%\right)$.

The recycling performance of ionic liquid has the most benefits from the point of view of environmental protection. During the reaction workup, the white product was precipitated out when ice water was added, after filtration, the mother liquid was evaporated in a vacuum, and ionic liquid was recovered and could be reused for three times. The results are presented in Table 2.

Table 2. Effect of ionic liquid recycling on Beckmann rearrangement.

\begin{tabular}{cccc}
\hline Reaction Turn & $\mathbf{P}_{\mathbf{2}} \mathbf{O}_{5} / \%$ & Conversion/\% & Yield/\% \\
\hline 1 & 8 & 91 & 91 \\
2 & 1 & 91 & 90 \\
3 & 0.5 & 90 & 88 \\
\hline
\end{tabular}

Reaction conditions: Benzophenone oxime $(9.5 \mathrm{mmol})$, [HMIm] $\mathrm{HSO}_{4}(11.4 \mathrm{mmol}), 90^{\circ} \mathrm{C}, 6 \mathrm{~h}$; Conversion was determined by Gas Chromatography (GC) using internal standard method.

In order to explore the scope and limitations of this reaction, we extended the procedure to various aryl-substituted and alkyl-substituted ketoximes. In general, the reaction proceeded easily under the best conditions and the amide products were isolated in excellent yields and high purity. The results are presented in Table 3.

Table 3. Formation of amides (3a-3o) from ketoxime (2a-2o) in the presence of ionic liquid and co-catalyst $\mathrm{P}_{2} \mathrm{O}_{5}$.

\begin{tabular}{ccc}
\hline Compd. & $R^{2}$ & Yield \% \\
\hline $3 a$ & & 91 \\
\hline $3 b$ & & 90 \\
\hline
\end{tabular}


Table 3. Cont.

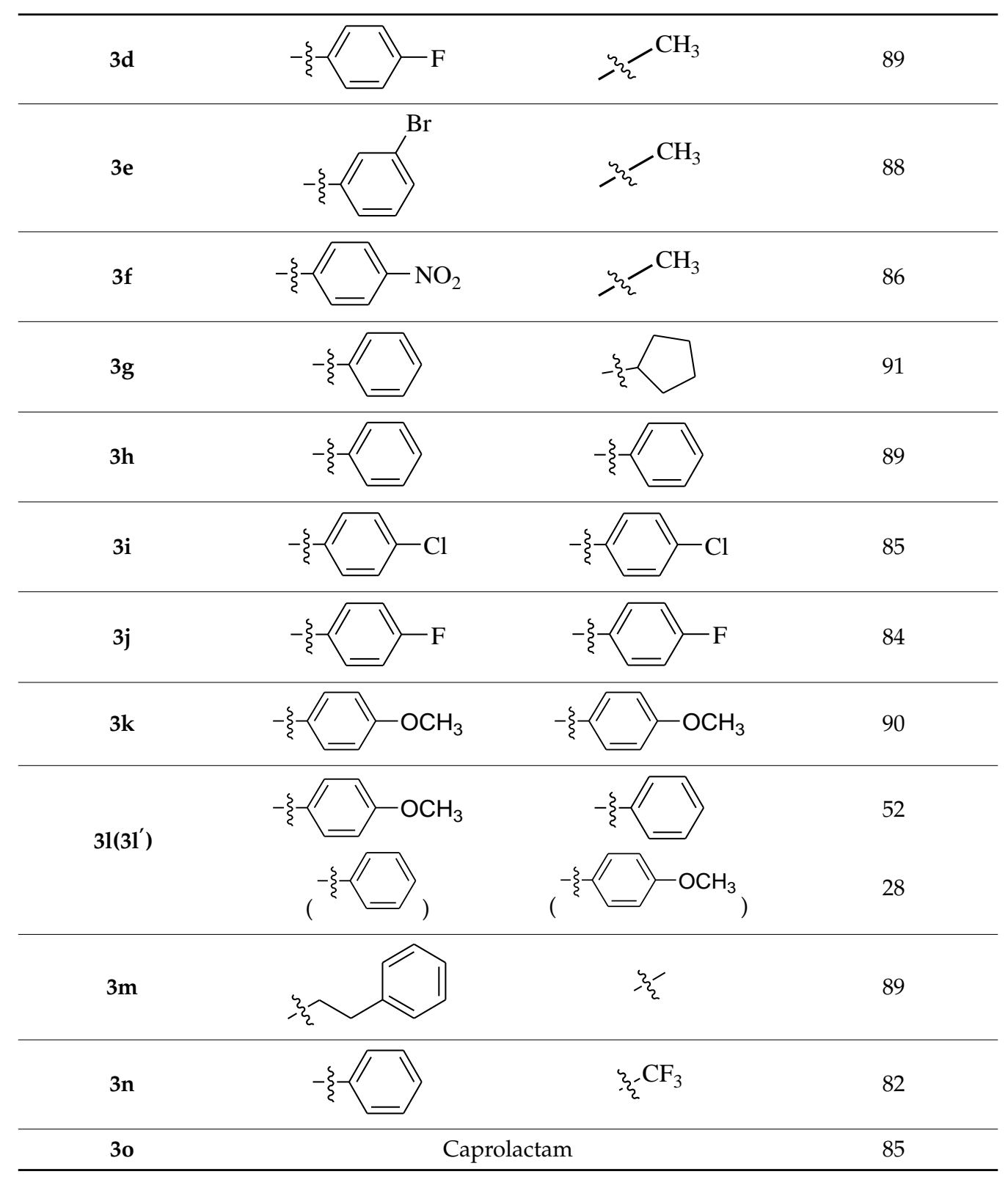

Reaction conditions: Benzophenone oxime $(9.5 \mathrm{mmol}),\left[\mathrm{HMIm}^{\circ} \mathrm{HSO}_{4}(11.4 \mathrm{mmol})\right.$, catalyst $\left(\mathrm{P}_{2} \mathrm{O}_{5} 8 \%\right), 90{ }^{\circ} \mathrm{C}, 6 \mathrm{~h}$.

\section{Experimental Section}

All melting points were determined using a YRT-3 Digital Melting Point Apparatus (Tianjin, China). All melting points were uncorrected. All new compounds were characterized by HRMS-EI(M+), ${ }^{1} \mathrm{H}$ and ${ }^{13} \mathrm{C}-\mathrm{NMR}$ spectra were recorded in $\mathrm{CDCl}_{3}$ or DMSO- $\mathrm{d}_{6}$ on a Bruker AV $600 \mathrm{MHz}$ or Bruker AV $400 \mathrm{MHz}$ instrument. HRMS spectra were obtained on an Agilent 6230 mass spectrometer.

\subsection{Synthesis of N-methyl-imidazolium Hydrosulfate $\left([\mathrm{HMIm}] \mathrm{HSO}_{4}\right)$}

$\mathrm{N}$-Methylimidazole $(8.2 \mathrm{~g}, 0.10 \mathrm{~mol})$ was cooled down to $0{ }^{\circ} \mathrm{C}$ and concentrated sulfuric acid $(10.0 \mathrm{~g})$ was added dropwise. After addition, the solution was stirred $24 \mathrm{~h}$ at room temperature, a transparent viscous liquid (17.6 g) was obtained. Yield: 99\%; IR $\left(\mathrm{cm}^{-1}\right)$ : 3345, 3150, 2870, 1447, 1337, $1221,1048,1082,887$. 


\subsection{General Procedures for Synthesis of Oxime Substrates $\mathbf{2 a - 2 o}$}

Ketone $(0.027 \mathrm{~mol})$ and hydroxylamine hydrochloride $(3.0 \mathrm{~g}, 0.043 \mathrm{~mol})$ were dissolved in EtOH $(10 \mathrm{~mL})$ and $\mathrm{H}_{2} \mathrm{O}(20 \mathrm{~mL})$. To the mixture was added $\mathrm{NaOH}(5.5 \mathrm{~g}, 0.137 \mathrm{~mol})$. The reaction mixture was heated under reflux and the reaction was monitored by thin layer chromatography (TLC). After completion of the reaction, the reaction mixture was cooled down to room temperature, to the reaction mixture were added concentrated hydrochloric acid $(15 \mathrm{~mL})$ and water $(100 \mathrm{~mL})$. The solid was filtered off and recrystallized from $\mathrm{EtOH}$, affording the products $\mathbf{2 a - 2 o . ~}$

2a: White solid, Yield: 91\%. m.p.: 87.6-88.7 ${ }^{\circ} \mathrm{C} ;{ }^{1} \mathrm{H}-\mathrm{NMR}\left(400 \mathrm{MHz}, \mathrm{CDCl}_{3}\right) \delta 7.60(\mathrm{~d}, J=8.9 \mathrm{~Hz}, 2 \mathrm{H})$, $6.93(\mathrm{~d}, J=8.9 \mathrm{~Hz}, 2 \mathrm{H}), 3.85(\mathrm{~s}, 3 \mathrm{H}), 2.30(\mathrm{~s}, 3 \mathrm{H}) ;{ }^{13} \mathrm{C}-\mathrm{NMR}\left(100 \mathrm{MHz}, \mathrm{CDCl}_{3}\right) \delta 160.5,155.6,129.0$, $127.4,113.9,55.3,13.3$.

2b: White solid, Yield: 93\%. m.p.: 88.0-89.0 ${ }^{\circ} \mathrm{C} ;{ }^{1} \mathrm{H}-\mathrm{NMR}\left(400 \mathrm{MHz}, \mathrm{CDCl}_{3}\right) \delta 7.55(\mathrm{~d}, J=8.2 \mathrm{~Hz}, 2 \mathrm{H})$, $7.22(\mathrm{~d}, J=8.0 \mathrm{~Hz}, 2 \mathrm{H}), 2.39(\mathrm{~s}, 3 \mathrm{H}), 2.31(\mathrm{~s}, 3 \mathrm{H}) ;{ }^{13} \mathrm{C}-\mathrm{NMR}\left(100 \mathrm{MHz}, \mathrm{CDCl}_{3}\right) \delta$ 156.0, 139.3, 133.7, 129.3, $126.0,21.3,12.3$.

2c: White solid, Yield: 90\%. m.p.: 51.7-53.0 ${ }^{\circ} \mathrm{C} ;{ }^{1} \mathrm{H}-\mathrm{NMR}\left(600 \mathrm{MHz}, \mathrm{CDCl}_{3}\right) \delta 7.68-7.64(\mathrm{~m}, 2 \mathrm{H})$, 7.44-7.40 (m, 3H), 2.35(s, 3H); ${ }^{13} \mathrm{C}-\mathrm{NMR}\left(150 \mathrm{MHz}, \mathrm{CDCl}_{3}\right) \delta 156.1,136.5,129.3,128.5,126.1,12.3$.

2d: White solid, Yield: 70\%. m.p.: 74.0-76.0 ${ }^{\circ} \mathrm{C} ;{ }^{1} \mathrm{H}-\mathrm{NMR}\left(600 \mathrm{MHz}, \mathrm{DMSO}-d_{6}\right) \delta 11.21$ (s, 1H), 7.76-7.59 $(\mathrm{m}, 2 \mathrm{H}), 7.22-7.19(\mathrm{~m}, 2 \mathrm{H}), 2.14(\mathrm{~s}, 3 \mathrm{H}) ;{ }^{13} \mathrm{C}-\mathrm{NMR}\left(150 \mathrm{MHz}, \mathrm{DMSO}-d_{6}\right) \delta 162.4\left(\mathrm{~d},{ }^{1} J_{\mathrm{CF}}=245.5 \mathrm{~Hz}\right)$, $152.1,133.5\left(\mathrm{~d},{ }^{4} J_{\mathrm{CF}}=3.0 \mathrm{~Hz}\right), 127.6\left(\mathrm{~d},{ }^{3} J_{\mathrm{CF}}=8.3 \mathrm{~Hz}\right), 115.2\left(\mathrm{~d},{ }^{2} J_{\mathrm{CF}}=21.5 \mathrm{~Hz}\right), 11.9$.

2e: White solid, Yield: 91\%. m.p.: 141.0-142.0 ${ }^{\circ} \mathrm{C} ;{ }^{1} \mathrm{H}-\mathrm{NMR}\left(600 \mathrm{MHz}, \mathrm{DMSO}-d_{6}\right) \delta 11.40(\mathrm{~s}, 1 \mathrm{H}), 7.80$ $(\mathrm{t}, J=1.8 \mathrm{~Hz}, 1 \mathrm{H}), 7.64(\mathrm{~d}, J=7.8 \mathrm{~Hz}, 1 \mathrm{H}), 7.55(\mathrm{~d}, J=7.8 \mathrm{~Hz}, 1 \mathrm{H}), 7.34(\mathrm{dd}, J=11.1,4.6 \mathrm{~Hz}, 1 \mathrm{H}), 2.14(\mathrm{~s}$, $3 \mathrm{H}) ;{ }^{13} \mathrm{C}-\mathrm{NMR}\left(150 \mathrm{MHz}, \mathrm{DMSO}-d_{6}\right) \delta 151.9,139.3,131.3,130.6,128.1,124.6,121.8,11.4$.

2f: Yellow solid, Yield: 90\%. m.p.: $172.0-173.0{ }^{\circ} \mathrm{C} ;{ }^{1} \mathrm{H}-\mathrm{NMR}\left(400 \mathrm{MHz}, \mathrm{DMSO}-d_{6}\right) \delta 11.78(\mathrm{~s}, 1 \mathrm{H})$, 8.28-8.18 (m, 2H), 7.96-7.88 (m, 2H), 2.21 (s, 3H); ${ }^{13}$ C-NMR (100 MHz, DMSO-d $\left.{ }_{6}\right) \delta 157.1,152.5,148.3$, $131.9,131.8,16.6$.

2g: White solid, Yield: 95.6\%. m.p.: $129.6-131.0{ }^{\circ} \mathrm{C} ;{ }^{1} \mathrm{H}-\mathrm{NMR}\left(600 \mathrm{MHz}, \mathrm{DMSO}-d_{6}\right) \delta 10.49(\mathrm{~s}, 1 \mathrm{H})$, 7.42-7.27 (m, 5H), 3.04-2.85 (m, 1H), 1.75-1.66 (m, 2H), 1.64-1.50 (m, 6H); ${ }^{13} \mathrm{C}-\mathrm{NMR}(150 \mathrm{MHz}$, DMSO-d $\left.d_{6}\right) \delta 158.6,135.8,128.3,128.2,128.1,45.2,30.3,24.9$.

2h: White solid, Yield: 60\%. m.p.: 92.0-93.0 ${ }^{\circ} \mathrm{C} ;{ }^{1} \mathrm{H}-\mathrm{NMR}\left(600 \mathrm{MHz}, \mathrm{DMSO}-d_{6}\right) \delta 11.40$ (s, 1H), 7.48-7.44 $(\mathrm{m}, 2 \mathrm{H}), 7.43-7.40(\mathrm{~m}, 1 \mathrm{H}), 7.387 .37(\mathrm{~m}, 5 \mathrm{H}), 7.30-7.25(\mathrm{~m}, 2 \mathrm{H}) ;{ }^{13} \mathrm{C}-\mathrm{NMR}\left(150 \mathrm{MHz}, \mathrm{DMSO}-d_{6}\right) \delta 155.2$, $136.8,133.5,128.88,128.86,128.40,128.36,128.2,127.0$.

2i: White solid, Yield: 87.5\%. m.p.: $134.0-136.0{ }^{\circ} \mathrm{C} ;{ }^{1} \mathrm{H}-\mathrm{NMR}\left(600 \mathrm{MHz}, \mathrm{CDCl}_{3}\right) \delta 7.48(\mathrm{~d}, J=8.6 \mathrm{~Hz}$, 2H), 7.43-7.37 (m, 4H), 7.36-7.31 (m, 2H); ${ }^{13} \mathrm{C}-\mathrm{NMR}\left(150 \mathrm{MHz}, \mathrm{CDCl}_{3}\right) \delta 156.1,135.9,135.5,134.3,130.8$, $130.4,129.1,128.8,128.7$.

2j: White solid, Yield: 88.5\%. m.p.: 131.0-132.0 ${ }^{\circ} \mathrm{C} ;{ }^{1} \mathrm{H}-\mathrm{NMR}\left(600 \mathrm{MHz}, \mathrm{DMSO}-d_{6}\right) \delta 11.45(\mathrm{~s}, 1 \mathrm{H})$, 7.43-7.38 (m, 2H), 7.38-7.32 (m, 2H), $7.28(\mathrm{dd}, J=12.3,5.4 \mathrm{~Hz}, 2 \mathrm{H}), 7.19(\mathrm{dd}, \mathrm{J}=12.3,5.4 \mathrm{~Hz}, 2 \mathrm{H})$; ${ }^{13} \mathrm{C}-\mathrm{NMR}\left(150 \mathrm{MHz}, \mathrm{DMSO}-\mathrm{d}_{6}\right) \delta 162.6\left(\mathrm{~d},{ }^{1} J_{\mathrm{CF}}=244.6 \mathrm{~Hz}\right), 161.9\left(\mathrm{~d},{ }^{1} J_{\mathrm{CF}}=244.6 \mathrm{~Hz}\right), 153.4,133.2$, $131.3\left(\mathrm{~d},{ }^{3} J_{\mathrm{CF}}=8.2 \mathrm{~Hz}\right), 129.5,129.1\left(\mathrm{~d},{ }^{3} J_{\mathrm{CF}}=8.2 \mathrm{~Hz}\right), 115.4\left(\mathrm{~d},{ }^{2} J_{\mathrm{CF}}=22.6 \mathrm{~Hz}\right), 115.2\left(\mathrm{~d},{ }^{2} J_{\mathrm{CF}}=22.3 \mathrm{~Hz}\right)$.

2k: White solid, Yield: 87.5\%. m.p.: 129.0-130.0 ${ }^{\circ} \mathrm{C} ;{ }^{1} \mathrm{H}-\mathrm{NMR}\left(400 \mathrm{MHz}, \mathrm{DMSO}-d_{6}\right) \delta 11.1(\mathrm{~s}, 1 \mathrm{H}), 7.31$ $(\mathrm{d}, J=8.8 \mathrm{~Hz}, 2 \mathrm{H}), 7.24(\mathrm{~d}, J=8.8 \mathrm{~Hz}, 2 \mathrm{H}), 6.99(\mathrm{~d}, J=8.7 \mathrm{~Hz}, 2 \mathrm{H}), 6.91(\mathrm{~d}, J=8.8 \mathrm{~Hz}, 2 \mathrm{H}), 3.79(\mathrm{~s}, 3 \mathrm{H})$, $3.76(\mathrm{~s}, 3 \mathrm{H}) ;{ }^{13} \mathrm{C}-\mathrm{NMR}\left(100 \mathrm{MHz}, \mathrm{DMSO}-d_{6}\right) \delta 159.8,159.1,154.4,130.7,129.7,128.6,125.6,113.7,113.4$, $55.2,55.1$.

21: Light yellow solid, Yield: 87.9\%. m.p.:155.0-160.0 ${ }^{\circ} \mathrm{C}$; (isomer 1): ${ }^{1} \mathrm{H}-\mathrm{NMR}\left(600 \mathrm{MHz}, \mathrm{DMSO}-d_{6}\right)$ $\delta 11.27(\mathrm{~s}, 1 \mathrm{H}), 7.38-7.34(\mathrm{~m}, 4 \mathrm{H}), 7.29-7.24(\mathrm{~m}, 3 \mathrm{H}), 7.00(\mathrm{~d}, J=8.7 \mathrm{~Hz}, 2 \mathrm{H}), 3.79(\mathrm{~s}, 3 \mathrm{H})$; (isomer 2): ${ }^{1} \mathrm{H}-\mathrm{NMR}\left(600 \mathrm{MHz}, \mathrm{DMSO}-d_{6}\right) \delta 11.09(\mathrm{~s}, 1 \mathrm{H}), 7.46-7.43(\mathrm{~m}, 2 \mathrm{H}), 7.42-7.38(\mathrm{~m}, 3 \mathrm{H}), 7.30(\mathrm{~d}, J=8.8 \mathrm{~Hz}$, 
2H), $6.92(\mathrm{~d}, J=8.8 \mathrm{~Hz}, 2 \mathrm{H}), 3.75(\mathrm{~s}, 3 \mathrm{H})$; (isomer 1): ${ }^{13} \mathrm{C}-\mathrm{NMR}\left(100 \mathrm{MHz}, \mathrm{DMSO}-d_{6}\right) \delta 159.85,154.85$, $137.32,130.74,129.22,128.32,128.29,128.12,113.80,55.20$; (isomer 2$):{ }^{13} \mathrm{C}-\mathrm{NMR}\left(100 \mathrm{MHz}\right.$, DMSO- $\left.d_{6}\right) \delta$ $159.20,154.80,133.82,128.87,128.76,128.29,127.32,125.37,113.46,55.15$.

2m: Yield: $94.0 \%$. m.p.: $84-86{ }^{\circ} \mathrm{C} ;{ }^{1} \mathrm{H}-\mathrm{NMR}\left(600 \mathrm{MHz}, \mathrm{DMSO}-d_{6}\right) \delta 8.81$ (brs, $\left.1 \mathrm{H}\right), 7.30-7.18(\mathrm{~m}, 5 \mathrm{H})$, $2.83(\mathrm{t}, J=8.2 \mathrm{~Hz}, 2 \mathrm{H}), 2.51(\mathrm{t}, J=8.2 \mathrm{~Hz}, 2 \mathrm{H}), 1.91(\mathrm{~s}, 3 \mathrm{H}) ;{ }^{13} \mathrm{C}-\mathrm{NMR}\left(150 \mathrm{MHz}, \mathrm{DMSO}-d_{6}\right) \delta 157.9$, 141.0, 128.4, 128.3, 126.1, 37.7, 32.6,13.8.

2n: Yield: $90.0 \%$. m.p.: $79-81{ }^{\circ} \mathrm{C} ;{ }^{1} \mathrm{H}-\mathrm{NMR}\left(600 \mathrm{MHz}\right.$, DMSO- $\left.d_{6}\right) \delta 12.74(\mathrm{~s}, 1 \mathrm{H}), 7.73-7.16(\mathrm{~m}, 5 \mathrm{H})$; ${ }^{13} \mathrm{C}-\mathrm{NMR}\left(150 \mathrm{MHz}, \mathrm{DMSO}-d_{6}\right) \delta 145.2\left(\mathrm{q},{ }^{2} J_{\mathrm{CF}}=30.0\right), 130.6,129.0,128.9,127.2,121.6\left(\mathrm{q},{ }^{1} J_{\mathrm{CF} 3}=271.5\right)$.

2o: Yield: 85.5\%. m.p.: 89-91 ${ }^{\circ} \mathrm{C} ;{ }^{1} \mathrm{H}-\mathrm{NMR}\left(600 \mathrm{MHz}\right.$, DMSO- $\left.d_{6}\right) \delta 2.48(\mathrm{dd}, J=6.8,5.3,2 \mathrm{H}), 2.48(\mathrm{~m}$, 2H), 1.76-1.45 (m, 6H); ${ }^{13} \mathrm{C}-\mathrm{NMR}\left(150 \mathrm{MHz}, \mathrm{DMSO}_{6}\right) \delta$ 157.1, 31.6, 26.6, 25.4, 25.2, 23.8.

\subsection{General Procedures for the Synthesis of Amides $\mathbf{3 a}-\mathbf{3 o}$}

To a solution of the oxime substrates $\mathbf{2 a}-\mathbf{2 o}(9.50 \mathrm{mmol})$ in $(\mathrm{HMIm}) \mathrm{HSO}_{4}(2.05 \mathrm{~g}, 11.4 \mathrm{mmol})$, the co-catalyst $\mathrm{P}_{2} \mathrm{O}_{5}(0.15 \mathrm{~g}, 1.0 \mathrm{mmol})$ was added. Then the solution was heated to $90{ }^{\circ} \mathrm{C}$ and the reaction was monitored by TLC. After completion of the reaction, the mixture was extracted with ethyl acetate $(50 \mathrm{~mL})$ twice, and the combined organic phase was washed with the aqueous solution of sodium bicarbonate and brine, dried over anhydrous $\mathrm{Na}_{2} \mathrm{SO}_{4}$ and concentrated in vacuo to afford a residue, which was purified by column (ethyl acetate: petroleum ether $=1: 4$ ) to afford the products $\mathbf{3 a}-\mathbf{3 o}$.

3a [22]: White solid, Yield: $91 \%$. m.p.: $127.0-128.0{ }^{\circ} \mathrm{C} ;{ }^{1} \mathrm{H}-\mathrm{NMR}\left(600 \mathrm{MHz}, \mathrm{DMSO}-d_{6}\right) \delta 9.80$ (brs, $1 \mathrm{H}), 7.51-7.46(\mathrm{~m}, 2 \mathrm{H}), 7.20(\mathrm{~d}, J=7.9 \mathrm{~Hz}, 1 \mathrm{H}), 6.89-6.84(\mathrm{~m}, 2 \mathrm{H}), 3.71(\mathrm{~s}, 3 \mathrm{H}), 2.01(\mathrm{~s}, 3 \mathrm{H}) ;{ }^{13} \mathrm{C}-\mathrm{NMR}$ $\left(150 \mathrm{MHz}, \mathrm{DMSO}-d_{6}\right) \delta 168.2,155.5,133.0,121.0,114.2,55.6,24.3$; HRMS(+): calcd. for $\mathrm{C}_{9} \mathrm{H}_{11} \mathrm{NO}_{2}$ $[\mathrm{M}+\mathrm{H}]^{+} 166.0863$, found 166.0859; calcd. for $\mathrm{C}_{9} \mathrm{H}_{11} \mathrm{NO}_{2} \mathrm{Na}[\mathrm{M}+\mathrm{Na}]^{+} 188.0682$, found 188.0682.

3b [22]: White solid, Yield: 90\%. m.p.: 149.0-150.0 ${ }^{\circ} \mathrm{C} ;{ }^{1} \mathrm{H}-\mathrm{NMR}\left(600 \mathrm{MHz}, \mathrm{DMSO}-d_{6}\right) \delta 9.84(\mathrm{~s}, 1 \mathrm{H})$, $7.46(\mathrm{~d}, J=8.3 \mathrm{~Hz}, 2 \mathrm{H}), 7.09(\mathrm{~d}, J=8.3 \mathrm{~Hz}, 2 \mathrm{H}), 2.24(\mathrm{~s}, 3 \mathrm{H}), 2.02(\mathrm{~s}, 3 \mathrm{H}) ;{ }^{13} \mathrm{C}-\mathrm{NMR}\left(150 \mathrm{MHz}, \mathrm{DMSO}-d_{6}\right)$ $\delta 173.2,142.1,137.0,134.1,124.2,29.2,25.6$; HRMS(+): calcd. for $\mathrm{C}_{9} \mathrm{H}_{11} \mathrm{NO}[\mathrm{M}+\mathrm{H}]^{+} 150.0913$, found 150.0912; calcd. for $\mathrm{C}_{9} \mathrm{H}_{11} \mathrm{NONa}[\mathrm{M}+\mathrm{Na}]^{+} 172.0733$, found 172.0741 .

3c [23]: White solid, Yield: $90 \%$. m.p.: $108.5-110.0{ }^{\circ} \mathrm{C} ;{ }^{1} \mathrm{H}-\mathrm{NMR}\left(600 \mathrm{MHz}, \mathrm{DMSO}-d_{6}\right) \delta 9.93$ (brs, $\left.1 \mathrm{H}\right)$, $7.58(\mathrm{dd}, J=1.0,8.5 \mathrm{~Hz}, 2 \mathrm{H}), 7.29(\mathrm{dd}, J=7.5,8.4 \mathrm{~Hz}, 2 \mathrm{H}), 7.08-6.91(\mathrm{~m}, 1 \mathrm{H}), 2.05(\mathrm{~s}, 3 \mathrm{H}) ;{ }^{13} \mathrm{C}-\mathrm{NMR}$ $\left(150 \mathrm{MHz}, \mathrm{DMSO}-d_{6}\right) \delta 168.7,139.8,129.1,123.4,119.4,24.5$; $\mathrm{HRMS}(+)$ : calcd. for $\mathrm{C}_{8} \mathrm{H}_{9} \mathrm{NO}[\mathrm{M}+\mathrm{H}]^{+}$ 136.0757, found 136.0755; calcd. for $\mathrm{C}_{8} \mathrm{H}_{9} \mathrm{NONa}[\mathrm{M}+\mathrm{Na}]^{+}$158.0576, found 158.0572.

3d [24]: Light yellow solid, Yield: $89 \%$. m.p.: $153-155^{\circ} \mathrm{C} ;{ }^{1} \mathrm{H}-\mathrm{NMR}\left(600 \mathrm{MHz}\right.$, DMSO- $\left.d_{6}\right) \delta 9.99$ (brs, 1H), 7.87-7.47 (m, 2H), $7.12(\mathrm{t}, J=8.99 \mathrm{~Hz}, 2 \mathrm{H}), 2.04(\mathrm{~s}, 3 \mathrm{H}) ;{ }^{13} \mathrm{C}-\mathrm{NMR}\left(150 \mathrm{MHz}, \mathrm{DMSO}-d_{6}\right) \delta 168.6$, 158.3.8 $\left(\mathrm{d},{ }^{1} J_{\mathrm{CF}}=237.0 \mathrm{~Hz}\right), 136.2\left(\mathrm{~d},{ }^{4} J_{\mathrm{CF}}=1.5 \mathrm{~Hz}\right), 121.1\left(\mathrm{~d},{ }^{3} J_{\mathrm{CF}}=7.5 \mathrm{~Hz}\right), 115.2\left(\mathrm{~d},{ }^{2} J_{\mathrm{CF}}=22.5 \mathrm{~Hz}\right)$, 24.3; HRMS(+): calcd. for $\mathrm{C}_{8} \mathrm{H}_{8} \mathrm{FNO}[\mathrm{M}+\mathrm{H}]^{+}$154.0663, found 154.0665; calcd. for $\mathrm{C}_{8} \mathrm{H}_{8} \mathrm{FNONa}$ $[\mathrm{M}+\mathrm{Na}]^{+}$176.0482, found 176.0481 .

3e [25]: White solid, Yield: 88\%. m.p.: 87.0-89.0 ${ }^{\circ} \mathrm{C}$; ${ }^{1} \mathrm{H}-\mathrm{NMR}\left(600 \mathrm{MHz}, \mathrm{DMSO}-d_{6}\right) \delta 10.11$ (brs, $1 \mathrm{H}), 7.95(\mathrm{~s}, 1 \mathrm{H}), 7.47(\mathrm{~d}, J=7.9 \mathrm{~Hz}, 1 \mathrm{H}), 7.27-7.23(\mathrm{~m}, 1 \mathrm{H}), 7.22-7.19(\mathrm{~m}, 1 \mathrm{H}), 2.05(\mathrm{~s}, 3 \mathrm{H}) ;{ }^{13} \mathrm{C}-\mathrm{NMR}$ $\left(150 \mathrm{MHz}, \mathrm{CDCl}_{3}\right) \delta 169.1,141.3,131.1,126.0,122.0,121.7,118.1,24.5 ; \mathrm{HRMS}(+)$ : calcd. for $\mathrm{C}_{8} \mathrm{H}_{8} \mathrm{BrNO}$ $[\mathrm{M}+\mathrm{H}]^{+}$213.9862, found 213.9860; calcd. for $\mathrm{C}_{8} \mathrm{H}_{8} \mathrm{BrNONa}[\mathrm{M}+\mathrm{Na}]^{+} 235.9681$, found 235.9681 .

3f [25]: Yellow solid, Yield: 86\%. m.p.: $214.0-215.0{ }^{\circ} \mathrm{C} ;{ }^{1} \mathrm{H}-\mathrm{NMR}\left(600 \mathrm{MHz}, \mathrm{DMSO}-d_{6}\right) \delta 10.57(\mathrm{~s}, 1 \mathrm{H})$, $8.21(\mathrm{~d}, J=9.2 \mathrm{~Hz}, 2 \mathrm{H}), 7.82(\mathrm{~d}, J=9.4 \mathrm{~Hz}, 2 \mathrm{H}), 2.12(\mathrm{~s}, 3 \mathrm{H}) ;{ }^{13} \mathrm{C}-\mathrm{NMR}\left(150 \mathrm{MHz}\right.$, DMSO- $\left.d_{6}\right) \delta 169.8$, 145.9, 142.3, 125.4, 119.0, 24.7; HRMS(+): calcd. for $\mathrm{C}_{8} \mathrm{H}_{8} \mathrm{~N}_{2} \mathrm{O}_{3}[\mathrm{M}+\mathrm{H}]^{+}$181.0608, found181.0610; Calcd. for $\mathrm{C}_{8} \mathrm{H}_{8} \mathrm{~N}_{2} \mathrm{O}_{3} \mathrm{Na}[\mathrm{M}+\mathrm{Na}]^{+}$203.0433, found 203.0437.

3g: White solid, Yield: $91 \%$. m.p.: $160.0-161.0{ }^{\circ} \mathrm{C} ;{ }^{1} \mathrm{H}-\mathrm{NMR}\left(600 \mathrm{MHz}, \mathrm{DMSO}-d_{6}\right) \delta 8.29(\mathrm{~d}, J=7.0 \mathrm{~Hz}$, $1 \mathrm{H}), 7.88-7.80(\mathrm{~m}, 2 \mathrm{H}), 7.54-7.49(\mathrm{~m}, 1 \mathrm{H}), 7.48-7.42(\mathrm{~m}, 2 \mathrm{H}), 4.46-3.96(\mathrm{~m}, 1 \mathrm{H}), 1.96-1.81(\mathrm{~m}, 2 \mathrm{H})$, 
1.74-1.64 (m, 2H), 1.60-1.43 (m, 4H); ${ }^{13} \mathrm{C}-\mathrm{NMR}\left(150 \mathrm{MHz}, \mathrm{DMSO}-d_{6}\right) \delta$ 166.4, 135.3, 131.4, 128.6, 127.7, 51.4, 32.6, 24.1; HRMS(+): calcd. for $\mathrm{C}_{12} \mathrm{H}_{15} \mathrm{NO}[\mathrm{M}+\mathrm{H}]^{+}$190.1226, found 190.1228; calcd. for $\mathrm{C}_{12} \mathrm{H}_{15} \mathrm{NO} \mathrm{Na}[\mathrm{M}+\mathrm{Na}]^{+}$212.1046, found 212.1044.

3h [23]: White solid, Yield: 89\%. m.p.: $162.6-163.0{ }^{\circ} \mathrm{C} ;{ }^{1} \mathrm{H}-\mathrm{NMR}\left(400 \mathrm{MHz}, \mathrm{DMSO}-d_{6}\right) \delta 10.25(\mathrm{~s}, 1 \mathrm{H})$, 7.99-7.92 (m, 2H), $7.78(\mathrm{~d}, J=7.6 \mathrm{~Hz}, 2 \mathrm{H}), 7.63-7.58(\mathrm{~m}, 1 \mathrm{H}), 7.57-7.51(\mathrm{~m}, 2 \mathrm{H}), 7.36(\mathrm{t}, J=7.9 \mathrm{~Hz}, 2 \mathrm{H})$, 7.15-7.07 (m, 1H); ${ }^{13} \mathrm{C}-\mathrm{NMR}\left(100 \mathrm{MHz}, \mathrm{DMSO}-d_{6}\right) \delta 166.0,139.6,135.5,132.0,129.1,128.9,128.1,124.1$, 120.8; HRMS(+): calcd. for $\mathrm{C}_{13} \mathrm{H}_{11} \mathrm{NO}[\mathrm{M}+\mathrm{H}]^{+} 198.0913$, found 198.0913; calcd. for $\mathrm{C}_{13} \mathrm{H}_{11} \mathrm{NONa}$ $[\mathrm{M}+\mathrm{Na}]^{+}$220.0733, found 220.0733.

3i [25]: White solid, Yield: 85\%. m.p.: $210.0-212.0{ }^{\circ} \mathrm{C} ;{ }^{1} \mathrm{H}-\mathrm{NMR}\left(600 \mathrm{MHz}, \mathrm{DMSO}-d_{6}\right) \delta 10.45$ (s, $1 \mathrm{H}), 8.02-7.96(\mathrm{~m}, 2 \mathrm{H}), 7.86-7.79(\mathrm{~m}, 2 \mathrm{H}), 7.66-7.59(\mathrm{~m}, 2 \mathrm{H}), 7.45-7.39(\mathrm{~m}, 2 \mathrm{H}) ;{ }^{13} \mathrm{C}-\mathrm{NMR}(150 \mathrm{MHz}$, DMSO- $\left.d_{6}\right) \delta 165.0,138.4,137.0,133.8,130.1,129.0,127.9,122.4$; HRMS(+): calcd. for $\mathrm{C}_{13} \mathrm{H}_{9} \mathrm{C}_{12} \mathrm{NO}$ $[\mathrm{M}+\mathrm{H}]^{+}$266.0134, found 266.0129; calcd. for $\mathrm{C}_{13} \mathrm{H}_{9} \mathrm{C}_{12} \mathrm{NONa}[\mathrm{M}+\mathrm{Na}]^{+} 287.9953$, found 287.9951 .

3j [26]: Light yellow solid, Yield: 84\%. m.p.: 183-185.0 ${ }^{\circ} \mathrm{C} ;{ }^{1} \mathrm{H}-\mathrm{NMR}\left(600 \mathrm{MHz}, \mathrm{DMSO}-d_{6}\right) \delta 10.31$ (s, $1 \mathrm{H}), 8.04-8.02(\mathrm{~m}, 2 \mathrm{H}), 7.82-7.72(\mathrm{~m}, 2 \mathrm{H}), 7.37(\mathrm{t}, J=8.8 \mathrm{~Hz}, 2 \mathrm{H}), 7.19(\mathrm{t}, J=8.8 \mathrm{~Hz}, 2 \mathrm{H}) ;{ }^{13} \mathrm{C}-\mathrm{NMR}$ $\left(150 \mathrm{MHz}, \mathrm{DMSO}-d_{6}\right) \delta 164.3,164.1\left(\mathrm{~d},{ }^{1} J_{\mathrm{CF}}=249.5 \mathrm{~Hz}\right), 158.3\left(\mathrm{~d},{ }^{1} J_{\mathrm{CF}}=240.3 \mathrm{~Hz}\right), 135.4,131.2,130.4(\mathrm{~d}$, $\left.{ }^{3} J_{\mathrm{CF}}=9.0 \mathrm{~Hz}\right), 122.2\left(\mathrm{~d},{ }^{3} J_{\mathrm{CF}}=7.8 \mathrm{~Hz}\right), 115.3\left(\mathrm{~d},{ }^{2} J_{\mathrm{CF}}=22.4 \mathrm{~Hz}\right), 115.2\left(\mathrm{~d},{ }^{2} J_{\mathrm{CF}}=22.8 \mathrm{~Hz}\right) ; \mathrm{HRMS}(+)$ : calcd. for $\mathrm{C}_{13} \mathrm{H}_{9} \mathrm{~F}_{2} \mathrm{NO}[\mathrm{M}+\mathrm{H}]^{+} 234.0725$, found 234.0727; calcd. for $\mathrm{C}_{13} \mathrm{H}_{9} \mathrm{~F}_{2} \mathrm{NONa}[\mathrm{M}+\mathrm{Na}]^{+}$ 256.0544 , found 256.0546 .

3k [26]: Light yellow solid, Yield: $90 \%$. m.p.: $204.0-205.0{ }^{\circ} \mathrm{C} ;{ }^{1} \mathrm{H}-\mathrm{NMR}\left(600 \mathrm{MHz}, \mathrm{CDCl}_{3}\right) \delta 7.83$ (d, $J=8.8 \mathrm{~Hz}, 2 \mathrm{H}), 7.65(\mathrm{~s}, 1 \mathrm{H}), 7.52(\mathrm{~d}, J=9.0 \mathrm{~Hz}, 2 \mathrm{H}), 6.97(\mathrm{~d}, J=8.8 \mathrm{~Hz}, 2 \mathrm{H}), 6.91(\mathrm{~d}, J=9.0 \mathrm{~Hz}, 2 \mathrm{H})$, $3.87(\mathrm{~s}, 3 \mathrm{H}), 3.81(\mathrm{~s}, 3 \mathrm{H}) ;{ }^{13} \mathrm{C}-\mathrm{NMR}\left(150 \mathrm{MHz}, \mathrm{DMSO}-d_{6}\right) \delta 164.5,161.7,155.4,132.4,129.4,127.1,121.9$, 113.7, 113.5, 55.4, 55.2; $\mathrm{HRMS}(+)$ : calcd. for $\mathrm{C}_{15} \mathrm{H}_{15} \mathrm{NO}_{3}[\mathrm{M}+\mathrm{H}]^{+} 258.1125$, found 258.1127; calcd. for $\mathrm{C}_{15} \mathrm{H}_{15} \mathrm{NO}_{3} \mathrm{Na}[\mathrm{M}+\mathrm{Na}]^{+}$280.0944, found 280.0946.

31 [25] (N-(4-Methoxyphenyl)benzamide): Light yellow solid, Yield: 52\%. M.p.: 156.5-159.5 ${ }^{\circ} \mathrm{C} ;{ }^{1} \mathrm{H}-\mathrm{NMR}$ $\left(600 \mathrm{MHz}, \mathrm{DMSO}-d_{6}\right) \delta 10.07(\mathrm{~s}, 1 \mathrm{H}), 7.95(\mathrm{~d}, J=9.2 \mathrm{~Hz}, 2 \mathrm{H}), 7.74(\mathrm{~d}, J=7.6 \mathrm{~Hz}, 2 \mathrm{H}), 7.51(\mathrm{t}, J=7.6 \mathrm{~Hz}$, $1 \mathrm{H}), 7.33(\mathrm{t}, J=7.9 \mathrm{~Hz}, 2 \mathrm{H}), 7.05(\mathrm{~d}, J=9.2 \mathrm{~Hz}, 2 \mathrm{H}), 3.84(\mathrm{~s}, 3 \mathrm{H}) ;{ }^{13} \mathrm{C}-\mathrm{NMR}\left(100 \mathrm{MHz}\right.$, DMSO- $\left.d_{6}\right) \delta 164.94$, $161.91,139.36,131.40,129.61,128.58,123.45,120.37,113.62,55.45$; HRMS(+): calcd. for $\mathrm{C}_{14} \mathrm{H}_{13} \mathrm{NO}_{2}$ $[\mathrm{M}+\mathrm{H}]^{+}$228.1019, found 228.1020; calcd. for $\mathrm{C}_{14} \mathrm{H}_{13} \mathrm{NO}_{2} \mathrm{Na}[\mathrm{M}+\mathrm{Na}]^{+}$250.0838, found 250.0838; (4-Methoxy-N-phenylbenzamide): Light yellow solid, Yield: $28 \%{ }^{1} \mathrm{H}-\mathrm{NMR}\left(600 \mathrm{MHz}, \mathrm{DMSO}-d_{6}\right) \delta 10.12$ $(\mathrm{s}, 1 \mathrm{H}), 7.94(\mathrm{~d}, J=7.2 \mathrm{~Hz}, 2 \mathrm{H}), 7.67(\mathrm{~d}, J=9.0 \mathrm{~Hz}, 2 \mathrm{H}), 7.57(\mathrm{t}, J=7.2 \mathrm{~Hz}, 1 \mathrm{H}), 7.09(\mathrm{t}, J=8.8 \mathrm{~Hz}, 2 \mathrm{H})$, $6.93(\mathrm{~d}, J=9.0 \mathrm{~Hz}, 2 \mathrm{H}), 3.74(\mathrm{~s}, 3 \mathrm{H}) ;{ }^{13} \mathrm{C}-\mathrm{NMR}\left(100 \mathrm{MHz}, \mathrm{DMSO}-d_{6}\right) \delta 165.14,155.58,135.07,132.24$, 128.37, 127.56, 127.00, 122.02, 113.76, 55.20. HRMS(+): calcd. for $\mathrm{C}_{14} \mathrm{H}_{13} \mathrm{NO}_{2}[\mathrm{M}+\mathrm{H}]^{+} 228.1019$, found 228.1020; calcd. for $\mathrm{C}_{14} \mathrm{H}_{13} \mathrm{NO}_{2} \mathrm{Na}[\mathrm{M}+\mathrm{Na}]^{+} 250.0838$, found 250.0838 .

3m [27]: Yield: $89 \%$. m.p.: $113-114{ }^{\circ} \mathrm{C} ;{ }^{1} \mathrm{H}-\mathrm{NMR}\left(600 \mathrm{MHz}, \mathrm{DMSO}-d_{6}\right) \delta 7.34-7.30(\mathrm{~m}, 2 \mathrm{H}), 7.26-7.21$ $(\mathrm{m}, 3 \mathrm{H}), 3.85-3.77(\mathrm{~m}, 2 \mathrm{H}), 2.84-2.74(\mathrm{~m}, 2 \mathrm{H}), 2.29$ (s, 3H); ${ }^{13} \mathrm{C}-\mathrm{NMR}\left(150 \mathrm{MHz}, \mathrm{DMSO}-d_{6}\right) \delta 173.4$, 139.0, 129.3, 129.0, 126.9, 46.4, 34.8, 26.6. HRMS(+): calcd. for $\mathrm{C}_{10} \mathrm{H}_{13} \mathrm{NO}[\mathrm{M}+\mathrm{H}]^{+} 164.1070$, found 164.1071; calcd. for $\mathrm{C}_{10} \mathrm{H}_{13} \mathrm{NONa}[\mathrm{M}+\mathrm{Na}]^{+} 186.0889$, found 186.0884 .

3n [28]: Yield: $82 \%$. m.p.: $84-87{ }^{\circ} \mathrm{C} ;{ }^{1} \mathrm{H}-\mathrm{NMR}\left(600 \mathrm{MHz}, \mathrm{DMSO}-d_{6}\right) \delta 11.26$ (brs, $\left.1 \mathrm{H}\right), 7.70(\mathrm{dd}$, $J=0.83,8.53 \mathrm{~Hz}, 2 \mathrm{H}), 7.43-7.38(\mathrm{~m}, 2 \mathrm{H}), 7.24-7.20(\mathrm{~m}, 1 \mathrm{H}),{ }^{13} \mathrm{C}-\mathrm{NMR}\left(150 \mathrm{MHz}, \mathrm{DMSO}-d_{6}\right) \delta 155.0(\mathrm{q}$, $\left.{ }^{2} J_{\mathrm{CF}}=36.0\right), 136.8,129.4,126.0,121.5,116.3\left(\mathrm{q},{ }^{1} J_{\mathrm{CF} 3}=286.5\right)$. HRMS(+): calcd. for $\mathrm{C}_{8} \mathrm{H}_{6} \mathrm{~F}_{3} \mathrm{NO}[\mathrm{M}+\mathrm{H}]^{+}$ 190.0474, found 190.0472; calcd. for $\mathrm{C}_{8} \mathrm{H}_{6} \mathrm{~F}_{3} \mathrm{NONa}[\mathrm{M}+\mathrm{Na}]^{+} 212.0294$, found 212.0296.

3o [29]: Yield: $85 \%$. m.p.: $68-71{ }^{\circ} \mathrm{C} ;{ }^{1} \mathrm{H}-\mathrm{NMR}\left(600 \mathrm{MHz}\right.$, DMSO- $\left.d_{6}\right) \delta 7.41$ (brs, $\left.1 \mathrm{H}\right), 3.05$ (dd, $J=5.87$, $10.09 \mathrm{~Hz}, 2 \mathrm{H}), 2.38-2.13(\mathrm{~m}, 2 \mathrm{H}), 1.66(\mathrm{q}, J=5.87 \mathrm{~Hz}, 2 \mathrm{H}), 1.56-1.46(\mathrm{~m}, 4 \mathrm{H}) ;{ }^{13} \mathrm{C}-\mathrm{NMR}(150 \mathrm{MHz}$, DMSO- $\left.d_{6}\right) \delta 177.4,41.9,36.9,30.5,30.3,23.4$, HRMS(+): calcd. for $\mathrm{C}_{6} \mathrm{H}_{11} \mathrm{NO}[\mathrm{M}+\mathrm{H}]^{+} 114.0913$, found 114.0910; calcd. for $\mathrm{C}_{6} \mathrm{H}_{11} \mathrm{NONa}[\mathrm{M}+\mathrm{Na}]^{+}$136.0733, found136.0734. 


\section{Conclusions}

In conclusion, we successfully demonstrated an efficient approach for the synthesis of amide derivatives via Beckmann rearrangement of ketoxime by using Brønsted acidic ionic liquid $\mathrm{N}$-methyl-imidazolium hydrosulfate as an environmental friendly catalyst and solvent. The best reaction condition is: reaction temperature $90^{\circ} \mathrm{C}$, reaction time $6 \mathrm{~h}$, solvent $\mathrm{N}$-methyl-imidazolium hydrosulfate 10 grams, co-catalyst $\mathrm{P}_{2} \mathrm{O}_{5} 8 \mathrm{~mol} \%$. Ionic liquid can be reused three times. The procedure can be extended to various symmetrical and unsymmetrical aryl-substituted and alkyl-substituted ketoxime substrates. The aryl group migration products are sole products for unsymmetrical aryl alkyl substituted amides.

Author Contributions: H.H., X.Y., and S.Z. conceived and designed the experiments; X.C. and Z.X. performed the experiments; H.H. analyzed the data; and H.H. wrote the paper.

Funding: The project was supported by the Zhejiang province ecology first-class discipline and Zhejiang Provincial Department of Education general research projects (Y201636410).

Conflicts of Interest: The authors declare no conflict of interest.

\section{References}

1. Ghiaci, M.; Aghaei, H.; Oroojeni, M.; Aghabarari, B.; Rives, V.; Vicente, M.A.; Sobrados, I.; Sanz, J. Synthesis of paracetamol by liquid phase Beckmann rearrangement of 4-hydroxyacetophenone oxime over H3PO4/Al-MCM-41. Catal. Commun. 2009, 10, 1486-1492. [CrossRef]

2. $\quad$ Murray, W.V.; Lalan, P.; Gill, A.; Addo, M.F.; Lewis, J.M.; Lee, D.K.H.; Rampulla, R.; Wachter, M.P.; Hsi, J.D.; Underwood, D.C. Substituted piperidin-2-one biphenyltetrazoles as angiotensin II antagonists. Bioorg. Med. Chem. Lett. 1992, 2, 1775-1779. [CrossRef]

3. Constable, D.J.C.; Dunn, P.J.; Hayler, J.D.; Humphrey, G.R.; Leazer, J.J.L.; Linderman, R.J.; Lorenz, K.; Manley, J.; Pearlman, B.A.; Wells, A.; et al. Key green chemistry research areas-a perspective from pharmaceutical manufacturers. Green Chem. 2007, 9, 411-420. [CrossRef]

4. Chruma, J.J.; Cullen, D.J.; Bowman, L.; Toy, P.H. Polyunsaturated fatty acid amides from the Zanthoxylum genus-From culinary curiosities to probes for chemical biology. Nat. Prod. Rep. 2018, 35, 54-74. [CrossRef] [PubMed]

5. Feng, Q.; Liu, Z.-L.; Xiong, L.-X.; Wang, M.-Z.; Li, Y.-Q.; Li, Z.-M. Synthesis and Insecticidal Activities of Novel Anthranilic Diamides Containing Modified N-Pyridylpyrazoles. J. Agric. Food Chem. 2010, 58, 12327-12336. [CrossRef] [PubMed]

6. Zhenyu, J.; Ziqing, C.; Jianwen, C.; Li, Z.; Fan, Y.; Xianlang, Q.; Haifeng, B. High efficiency toughness of aromatic sulfonamide in polyamide 6. J. Appl. Polym. Sci. 2018, 135, 46527.

7. Nguyen, T.B.; Sorres, J.; Tran, M.Q.; Ermolenko, L.; Al-Mourabit, A. Boric Acid: A Highly Efficient Catalyst for Transamidation of Carboxamides with Amines. Org. Lett. 2012, 14, 3202-3205. [CrossRef] [PubMed]

8. Zhu, Y.-P.; Mampuys, P.; Sergeyev, S.; Ballet, S.; Maes, B.U.W. Amine Activation: N-Arylamino Acid Amide Synthesis from Isothioureas and Amino Acids. Adv. Synth. Catal. 2017, 359, 2481-2498. [CrossRef]

9. Thigulla, Y.; Ranga, S.; Ghosal, S.; Subbalakshmi, J.; Bhattacharya, A. One-Pot Two Step Nazarov-Schmidt Rearrangement for the Synthesis of Fused $\delta$-Lactam Systems. Chemistryselect 2017, 2, 9744-9750. [CrossRef]

10. Gao, P.; Bai, Z. Carbon Tetrabromide/Triphenylphosphine-Activated Beckmann Rearrangement of Ketoximes for Synthesis of Amides. Chin. J. Chem. 2017, 35, 1673-1677. [CrossRef]

11. Gregory, B.J.; Moodie, R.B.; Schofield, K. The Beckrnann Rearrangement of Acetophenone Oxirnes in Sulphuric Acid. Chem. Commun. 1968, 22, 1380-1381.

12. Maia, A.; Albanese, D.C.M.; Landini, D. Cyanuric chloride catalyzed Beckmann rearrangement of ketoximes in biodegradable ionic liquids. Tetrahedron 2012, 68, 1947-1950. [CrossRef]

13. De Luca, L.; Giacomelli, G.; Porcheddu, A. Beckmann Rearrangement of Oximes under Very Mild Conditions. J. Org. Chem. 2002, 67, 6272-6274. [CrossRef] [PubMed]

14. Furuya, Y.; Ishihara, K.; Yamamoto, H. Cyanuric Chloride as a Mild and Active Beckmann Rearrangement Catalyst. J. Am. Chem. Soc. 2005, 127, 11240-11241. [CrossRef] [PubMed] 
15. Blasco, T.; Corma, A.; Iborra, S.; Lezcano-González, I.; Montón, R. In situ multinuclear solid-state NMR spectroscopy study of Beckmann rearrangement of cyclododecanone oxime in ionic liquids: The nature of catalytic sites. J. Catal. 2010, 275, 78-83. [CrossRef]

16. Wang, B.; Gu, Y.; Luo, C.; Yang, T.; Yang, L.; Suo, J. Sulfamic acid as a cost-effective and recyclable catalyst for liquid Beckmann rearrangement, a green process to produce amides from ketoximes without waste. Tetrahedron Lett. 2004, 45, 3369-3372. [CrossRef]

17. Itoh, T. Activation of Lipase-Catalyzed Reactions Using Ionic Liquids for Organic Synthesis. Adv. Biochem. Eng. Biotechnol. 2018. [CrossRef]

18. Hallett, J.P.; Welton, T. Room-Temperature Ionic Liquids: Solvents for Synthesis and Catalysis. 2. Chem. Rev. 2011, 111, 3508-3576. [CrossRef] [PubMed]

19. Liu, S.; Xie, C.; Yu, S.; Liu, F. Dimerization of rosin using Brønsted-Lewis acidic ionic liquid as catalyst. Catal. Commun. 2008, 9, 2030-2034. [CrossRef]

20. Alvarez de Cienfuegos, L.; Robles, R.; Miguel, D.; Justicia, J.; Cuerva, J.M. Reduction Reactions in Green Solvents: Water, Supercritical Carbon Dioxide, and Ionic Liquids. ChemSusChem 2011, 4, 1035-1048. [CrossRef] [PubMed]

21. Singh, V.; Kaur, S.; Sapehiyia, V.; Singh, J.; Kad, G.L. Microwave accelerated preparation of [bmim][HSO4] ionic liquid: An acid catalyst for improved synthesis of coumarins. Catal. Commun. 2005, 6, 57-60. [CrossRef]

22. Mo, X.; Morgan, T.D.R.; Ang, H.T.; Hall, D.G. Scope and Mechanism of a True Organocatalytic Beckmann Rearrangement with a Boronic Acid/Perfluoropinacol System under Ambient Conditions. J. Am. Chem. Soc. 2018, 140, 5264-5271. [CrossRef] [PubMed]

23. Kore, R.; Srivastava, R. A simple, eco-friendly, and recyclable bi-functional acidic ionic liquid catalysts for Beckmann rearrangement. J. Mol. Catal. A Chem. 2013, 376, 90-97. [CrossRef]

24. Jeong, T.-S.; Kim, M.J.; Yu, H.; Kim, K.S.; Choi, J.-K.; Kim, S.-S.; Lee, W.S. (E)-Phenyl- and -heteroaryl-substituted O-benzoyl-(or acyl)oximes as lipoprotein-associated phospholipase A2 inhibitors. Bioorg. Med. Chem. Lett. 2005, 15, 1525-1527. [CrossRef] [PubMed]

25. Xie, F.K.; Du, C.; Pang, Y.D.; Lian, X.; Xue, C.T.; Chen, Y.Y.; Wang, X.F.; Cheng, M.S.; Guo, C.; Lin, B.; et al. Lewis acid-assisted $\mathrm{N}$-fluorobenzenesulfonimide-based electrophilic fluorine catalysis in Beckmann rearrangement. Tetrahedron Lett. 2016, 57, 5820-5824. [CrossRef]

26. Sharma, R.; Vishwakarma, R.A.; Bharate, S.B. Ligand-Free Copper-Manganese Spinel Oxide-Catalyzed Tandem One-Pot $\mathrm{C}-\mathrm{H}$ Amidation and N-Arylation of Benzylamines: A Facile Access to 2-Arylquinazolin-4(3H)-ones. Adv. Synth. Catal. 2016, 358, 3027-3033. [CrossRef]

27. Muraca, A.C.A.; Perecim, G.P.; Rodrigues, A.; Raminelli, C. Convergent Total Synthesis of (+/-)-Apomorphine via Benzyne Chemistry: Insights into the Mechanisms Involved in the Key Step. Synthesis 2017, 49, 3546-3557.

28. Schmidt, E.Y.; Ushakov, I.A.; Zorina, N.V.; Mikhaleva, A.I.; Trofimov, B.A. The reaction of 2-arylazo-1-vinylpyrroles with trifluoroacetic anhydride: Unexpected formation of N-aryl-2,2,2trifluoroacetamides and conjugated polymers. Mendeleev Commun. 2011, 21, 36-37. [CrossRef]

29. Xing, S.; Han, Q.; Shi, Z.; Wang, S.; Yang, P.; Wu, Q.; Li, M. A hydrophilic inorganic framework based on a sandwich polyoxometalate: Unusual chemoselectivity for aldehydes/ketones with in situ generated hydroxylamine. Dalton Trans. 2017, 46, 11537-11541. [CrossRef] [PubMed]

Sample Availability: Samples of the compounds are available from the authors. 